\title{
C3 Glomerulopathy and Therapeutic Potential of C5 Complement Inhibitors
}

\author{
Aysam Mahmoud1, Zeeshan Sheikh ${ }^{2 *}$, Safia Gilani' ${ }^{3}$ Paru Kathpalia ${ }^{4}$ \\ ${ }^{1}$ St James School of Medicine, The Quarter, Anguilla \\ ${ }^{2}$ Matrix Dynamics Group, Faculty of Dentistry, University of Toronto, Toronto, Canada \\ ${ }^{3}$ Department of Proteomics and HIV Research Lab, University of Southern California, Los Angeles, USA \\ ${ }^{4}$ Department of Nephrology, Chicago School of Medicine, University of Illinois, Chicago, USA \\ Email: "zeeshan.sheikh@utoronto.ca
}

Received 18 February 2016; accepted 25 March 2016; published 28 March 2016

Copyright (C) 2016 by authors and Scientific Research Publishing Inc.

This work is licensed under the Creative Commons Attribution International License (CC BY). http://creativecommons.org/licenses/by/4.0/

c) (i) Open Access

\begin{abstract}
C3 glomerulopathy is a disease including both dense deposit disease and C3 glomerulonephritis has an estimated prevalence of 2 to 3 per million. Originally, these pathologies were defined as glomerular pathology characterized by accumulation of $\mathrm{C} 3$ with absent or scanty immunoglobulin deposition. The keystone defect in both of these pathologies is the unregulated hyperactivity of alternative complement pathway. Specifically, in C3 glomerulopathy patients, there exists a prolongation of $\mathrm{C} 3$ cleavage which causes the uncontrolled alternative pathway activation. Many treatments have been investigated for treating C3 glomerulopathy to little or no avail, including calcineurin inhibitors, plasmapharesis, and anti-CD20 monoclonal antibodies. The next logical step is exploring the efficacy of anti-C5 monoclonal antibody therapy in $\mathrm{C} 3$ glomerulopathies to target the specific pathophysiology of this particular disease. Eculizumab is an anti-C5 monoclonal antibody that blocks the terminal step of complement activation. This drug has proven to be an effective treatment in other nephrologic pathologies that are caused by complement dysregulation. Here in this paper we discuss and present various case studies and clinical trials available that experiment with Eculizumab in patients with either dense deposit disease or C3 glomerulonephritis. In most of these patients, treatment with Eculizumab has demonstrated clinical and biochemical improvements in kidney function. These results provide encouraging evidence that suggest Eculizumab as a promising therapy for patients with $\mathrm{C} 3$ glomerulopathy and warrant that more extensive clinical trials can be designed as a next step.
\end{abstract}

\section{Keywords}

C3 Glomerulopathy, Dense Deposit Disease, C3 Glomerulopnephritis, MPGN II, Alternative Complement Pathway, Eculizumab, Proteinuria, Plasmapharesis, C5 Complement Therapy

\footnotetext{
${ }^{*}$ Corresponding author.
}

How to cite this paper: Mahmoud, A., Sheikh, Z., Gilani, S. and Kathpalia, P. (2016) C3 Glomerulopathy and Therapeutic Potential of C5 Complement Inhibitors. Open Journal of Nephrology, 6, 10-16. 


\section{Introduction}

The term C3 glomerulopathy is a pathological title that serves to include both dense deposit disease and C3 glomerulonephritis with an estimated prevalence of 2 to 3 per million population and usually diagnosed in childhood and young adulthood [1]. These pathologies are defined by C3 accumulation with absent or little immunoglobulin deposition. Most glomerular pathologies that involve complement deposits result from activation of the classic complement pathway by immune complexes of antigen and antibody [2]. This causes a secondary membranoproliferative glomerulonephritis (MPGN) caused by infection (lupus or hepatitis C) as well as idiopathic type 1 MPGN, acute post-infectious glomerulonephritis, and cyroglobulinemic glomerulonephritis [2]. All of these glomerular pathologies are characterized by the presence of both immunoglobulin (Ig) and complement on immunofluorescence staining of a renal biopsy [3]. However, as mentioned above, C3 glomerulopathy is proliferative glomerulonephritis characterized by the presence of $\mathrm{C} 3$ complement without detection of immunoglobulin [4]. This particular pathological pattern indicates a glomerulonephritis caused by inappropriate activation of the alternative complement pathway rather than the classic complement pathway. The alternative complement pathway is an antibody-independent mechanism of activating the complement cascade and leading to deposition of complement alone in the tissues [5]. Renal manifestations of C3 glomerulopathy are varied and non-specific, with hematuria, hypertension, proteinuria (including and in absence of nephrotic syndrome), and renal failure being reported [1] [3]. Non-renal clinical manifestations include acquired partial lipodystrophy, ocular drusen (lipoproteinaceous deposits of debris containing complement between the basal surfaces of retinal pigment endothelium), monoclonalgammopathy of undetermined significance and type 1 diabetes mellitus [1] [3] [5].

The nomenclature for dense deposit disease and C3 glomerulonephritis has been recently updated. Dense deposit disease was previously termed MPGN type II and was characterized by intramembranous electron dense material in the glomerular basement membrane [6]. C3 glomerulonephritis was previous known as idiopathic MPGN with isolated C3 deposits and was defined by subendothelial and/or mesangial electron dense deposits [2]. Both dense deposit disease and $\mathrm{C} 3$ glomerulonephritis show $\mathrm{C} 3$ deposition without simultaneous staining for immunoglobulin [7]. The lack of immunoglobulin incriminates the alternative complement pathway which can be inappropriately activated via many mechanisms. Mutations in various complement responsible proteins such as $\mathrm{C} 3$, factor $\mathrm{H}$, factor $\mathrm{I}$, and $\mathrm{C} 3$ can result in an uncontrolled alternative complement cascade as these proteins serve to inactivate that very pathway [8]. Acquired autoantibodies can also result in an overactive alternative complement pathway if those antibodies stabilize the activating complex (C3 nephritic factors) or if they affect the inhibitory complement factors such as factors I and H [5]. Regardless of the mechanism behind inappropriate activation of the alternative complement pathway, excess complement debris, including C3b breakdown products and components of the terminal complement cascade, is produced and they deposit in the glomerular basement membranes of the kidneys in high amounts.

Many therapies have been used relatively ineffectively in C3 glomerulopathy patients. Pharmacological therapies have included anti-cellular immune suppression therapy targeting $\mathrm{T}$ and/or B cells (i.e. cyclophosphamide, rituximab, or mycophenolate), glucocorticoids and plasma therapy [9]. Even renal transplantation has been proven an ineffective treatment for this disease [10]. A study consisting of 18 transplants in patients with dense deposit disease showed a $61 \%$ recurrence rate in the transplanted organ with subsequent decline in kidney function [11]. However, with an understanding of the specific pathophysiology of this disease, it is logical to state that therapies preventing the augmentation of the terminal complement cascade would be beneficial in deterring kidney damage. Data has increasingly shown that there is a causal relationship between the uncontrolled activation of the alternative pathway and $\mathrm{C} 3$ glomerulopathy [12]. With this understanding of the mechanism of the disease, anti-C5 therapy has potential to be an effective therapeutic approach as has been shown in certain animal models and anti-C5 therapy [11]. Eculizumab is a humanized monoclonal antibody that functions by interfering with the terminal step in complement activation [13] (Figure 1). It does this by binding with high affinity to $\mathrm{C} 5$ and thus preventing the production of the membrane attack complex that is necessary for the complement cascade. Eculizumab is an effective treatment in other conditions that are characterized by dysregulation of the complement system and is currently FDA approved for use in patients with paroxysmal nocturnal hemoglobinuria (PNH) and atypical hemolytic uremic syndrome (aHUS) [14] [15]. With the use of existing data from various case reports and a small clinical trial, Eculizumab shows promise to be utilized as an effective therapy in patients with $\mathrm{C} 3$ glomerulopathy. 


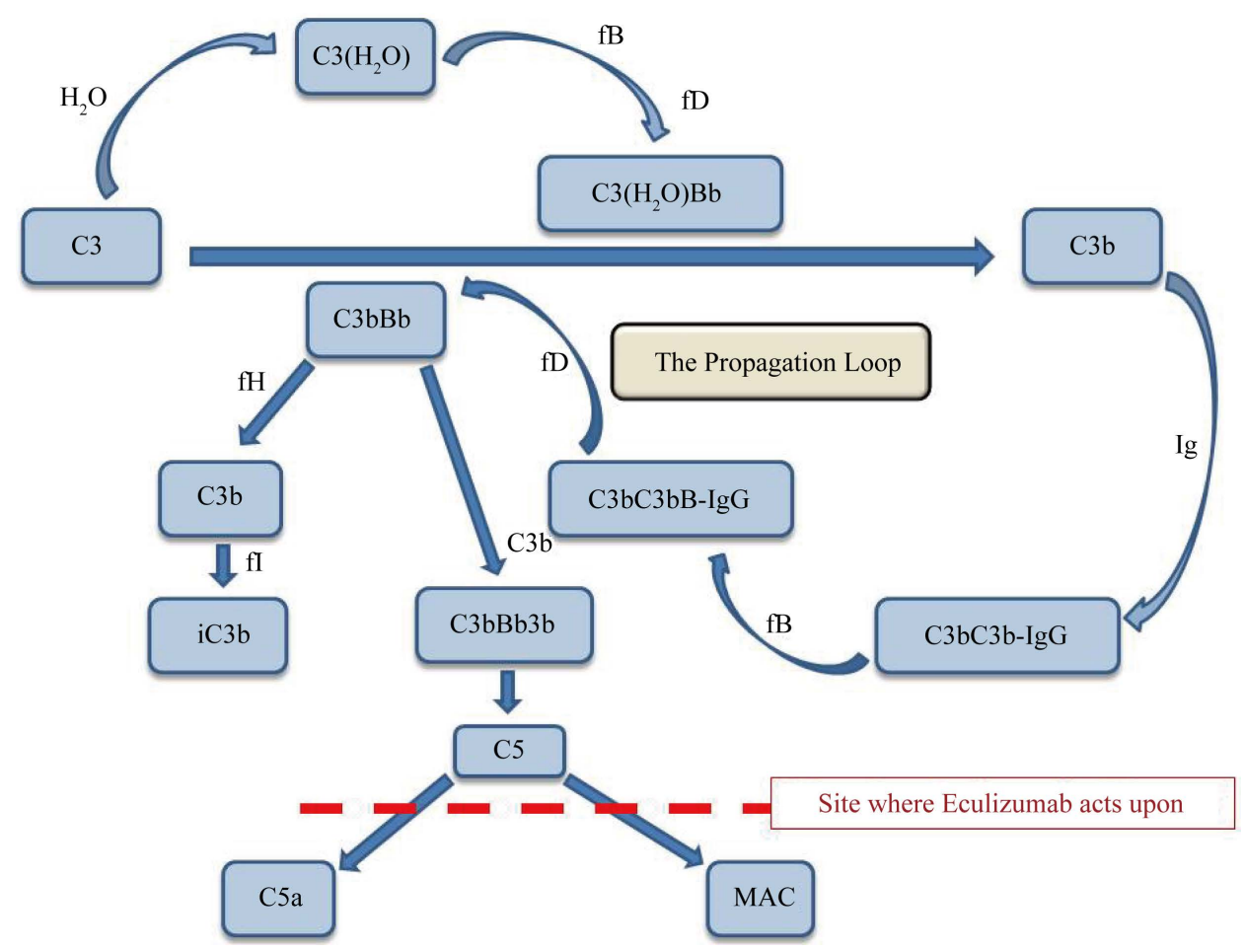

Figure 1. Schematic figure of the activation and regulation of the alternative complement pathway (C3: complement factor 3 nephritic factor; C5: complement factor 5; fB: complement factor B; fD: complement factor D and MAC: membrane attack complex). Adapted and redrawn from McCaughan et al. [13].

To investigate the effectiveness of $\mathrm{C} 5$ complement inhibitors in treating $\mathrm{C} 3$ glomerulopathy in this paper, we have used literature available on clinical trials and case reports associated with the efficacy of those drugs and relate the efficacy to the pathophysiological mechanism of the disease. Since there are not many clinical trials that have been conducted, we have included case studies that employ this pharmacotherapy. Included is data from patients with both dense deposit disease and $\mathrm{C} 3$ glomerulonephritis in which the diagnosis is confirmed by biopsy and renal status evaluated before and after the therapy. Coupled with this, we have discussed the pathophysiology of $\mathrm{C} 3$ Glomerulonephritis and explore why anti-C5 complement therapy is a promising therapeutic option to be explored further.

\section{Clinical Trials with Eculizumab}

The literature search revealed the availability of results from only one clinical trial that has been conducted. The clinical trial presented here is an open-label, proof of concept efficacy and safety study that included six patients [5]. The methodology used for the clinical trial is presented here with results discussed later in the discussion section of this paper. Three patients were diagnosed with dense deposit disease and three with C3 glomerulopathy. To be eligible for participation in this study, all six subjects were required to have undergone renal biopsy within 6 months of enrollment interpreted as either dense deposit disease or C3 glomerulopathy. Another eligibility requirement was that patients were required to have 24-hour urine protein greater than $1000 \mathrm{mg} / \mathrm{d}$, urine protein to creatinine ratio greater than 1.0, or acute kidney injury (defined as a $50 \%$ or greater increase in serum creatinine from baseline) at the time of enrollment. Exclusion criteria for this study included patient age less than 18 year, use of rituximab or any other monoclonal antibody within 6 months of the study, inability to discontinue use of other immunomodulatory medications (including cyclosporine, high-dose steroids, or mycophenolatemofetil), inability to taper prednisone administration to less than $10 \mathrm{mg} / \mathrm{d}$ if on chronic steroid therapy, other renal diseases (including diabetic nephropathy or renal vascular disease) and a baseline estimated GFR of less than $30 \mathrm{ml} / \mathrm{min}$ per $1.73 \mathrm{~m}^{2}[5]$.

All patients were treated with eculizumab every other week for one full year. No alterations were made to pa- 
tient renin-angiotensin-aldosterone system medications. Subjects were administered $900 \mathrm{mg}$ of eculizumab intravenously once per week for four weeks and then $1200 \mathrm{mg}$ intravenously on the fifth week and subsequently every other week afterward for a total of fifty three weeks. Laboratory measurements were taken every four weeks during the study and included basic metabolic panel, hepatic function panel, complete blood count, lipid profile, spot urine protein to creatinine ration, and serum complement levels ( $\mathrm{C} 3, \mathrm{C} 4$, and $\mathrm{CH} 50)$. Additionally, serum and plasma samples were sent every four weeks during treatment for functional testing of the alternative complement pathway. Finally, patients underwent a repeat renal biopsy at the one-year mark [5].

\section{Case Reports Showing the Use of Eculizumab}

To add to the one available clinical trial of Eculizumab therapy for $\mathrm{C} 3$ glomerulopathy, we will include results from three individual patient case reports regarding the effectiveness of Eculizumab in patients with the dense deposit disease variant of $\mathrm{C} 3$ glomerulopathy. A description of each case report is presented here with the results of Eculizumab therapy in each case report discussed later in the discussion section of this paper.

Daina et al. [16] presented the case of a female subject with long standing history of dense deposit disease and resulting nephrotic syndrome. This patient's glomerulopathy had since been unresponsive to five years of steroid therapy as well as treatment with Rituximab. She was started on Eculizumab at a dose of $900 \mathrm{mg}$ weekly for five weeks and then $1200 \mathrm{mg}$ every two weeks. Her response to treatment was measured via urinary protein and serum creatinine levels before and after treatment. Vivarelli et al. [17] reported another case of a 17-yearold patient with dense deposit disease, recurring proteinuria, microhematuria, and low serum C3 levels. Treatment with Eculizumab in this patient was evaluated with before and after measurements of proteinuria, plasma protein levels, and repeat renal biopsies 6 and 18 months after Eculizumab therapy started. Lastly, McCaughan et al. [13] demonstrates Eculizumab therapy in a 29-year-old patient with dense deposit disease post-renal transplant. This patient developed recurrence of her dense deposit disease four weeks after her renal transplant and showed subsequent proteinuria despite therapy with prednisone, tacrolimus, and mycophenolatemofetil. Thirteen weeks post-transplant, the patient was started on Eculizumab with a loading dose of $900 \mathrm{mg}$ on two separate occasions one week apart and then sustained with maintenance Eculizumab therapy of $600 \mathrm{mg}$ once every two weeks. Her response was measured via serum creatinine and urine protein levels.

\section{Discussion of Results from the Clinical Trial and Case Reports with Eculizumab}

The clinical trial performed by Bomback et al. [5] evaluating Eculizumab in six patients (three with dense deposit disease and three with $\mathrm{C} 3$ glomerulonephritis) suggested an overall response to eculizumab therapy in the majority of its subjects. Subject DDD1 (dense deposit disease) demonstrated an immediate and complete inhibition of the terminal complement cascade $(\mathrm{CH} 50=0)$ soon after Eculizumab therapy. This patient's serum creatinine also decreased from 1.8 to $1.5 \mathrm{mg} / \mathrm{dl}$ within 4 weeks of treatment. The subject's final creatinine measurement was in the $1.3-1.4 \mathrm{mg} / \mathrm{dl}$ range. His proteinuria levels were low throughout the duration of treatment. A repeat renal biopsy one year after initiation of therapy showed less activity with no evidence of endocapillary proliferation. To further emphasize the positive effect of Eculizumab on this patient's disease, four weeks after cessation of the drug, his creatinine rose to $1.5 \mathrm{mg} / \mathrm{dl}$, and then to $1.7 \mathrm{mg} / \mathrm{dl}$ after 8 weeks of treatment. The second subject with dense deposit disease (DDD2) did not necessarily show a clinical response to therapy, as both creatinine and proteinuria rose over 40 weeks of therapy. Because there was no clinical improvement, this patient withdrew from the study and thus no post therapy renal biopsy is available. Subject DDD3 experienced stable renal function throughout the course of the trial and after one year of treatment, proteinuria was measure between 0.2 to $1.9 \mathrm{~g} / \mathrm{g}$, and the serum albumin was stable above $4.0 \mathrm{~g} / \mathrm{dl}$. The patient's serum creatinine during the first 4 weeks of therapy was lower at $1.2 \mathrm{mg} / \mathrm{dl}$ than his baseline creatinine $(1.5-1.7 \mathrm{mg} / \mathrm{dl})$ measured over the previous twelve months before Eculizumab therapy. This subject's repeat renal biopsy showed decreased mesangial proliferation and less extensive deposits on electron microscopy.

The first patient with $\mathrm{C} 3$ Glomerulonephritis (C3GN1) experienced a flare of disease two months prior to starting this trial and as such was on high dose steroids and experiencing a higher than baseline serum creatinine and proteinuria. Once the steroids were adequately tapered off and the subject had been taking Eculizumab for twenty four weeks, renal function was stable, proteinuria declined from 2.3 to $0.5 \mathrm{~g} / \mathrm{g}$, and serum albumin increased from 3.2 to $3.9 \mathrm{~g} / \mathrm{dl}$. However, the patient showed worsening of kidney function over the subsequent twenty four weeks of therapy, demonstrated by a rise in creatinine to $2.4 \mathrm{mg} / \mathrm{dl}$, increased proteinuria of $1.8 \mathrm{~g} / \mathrm{g}$, 
and a decrease in albumin to $3.1 \mathrm{~g} / \mathrm{dl}$. In trend with these laboratory results, the repeat renal biopsy showed increased chronicity of disease in comparison with the pretreatment biopsy as demonstrated by $85 \%$ of glomeruli globally sclerotic (as compared to 50\% in previous biopsy). Subject C3GN2 had received a renal transplant four months prior and had been experiencing a steady rise in creatinine to $1.7-1.9 \mathrm{cg} / \mathrm{dl}$ as well as a rise in proteinuria from 0.1 to $1.4 \mathrm{~g} / \mathrm{g}$. However, throughout the study the patient demonstrated stable levels of serum creatinine, proteinuria, and serum albumin. Repeat renal biopsy post-therapy demonstrated less mesangial and endocapillary proliferation as well as a significantly decreased number of inflammatory cells in the glomeruli in comparison with the pre-treatment biopsy. The subject's renal function remained stable three weeks after Eculizumab was discontinued. However, at a seven week follow up after discontinuation of therapy, the patient's creatinine was found to be $8.4 \mathrm{mg} / \mathrm{dL}$. Repeat biopsy after this very high creatinine level showed recurrent active C3 Glomerulonephritis with crescents. This patient was restarted on Eculizumab post-trial and renal function greatly improved demonstrated by a creatinine of $3.8 \mathrm{mg} / \mathrm{dL}$ nine weeks after restarting therapy. The last patient with C3 Glomerulonephritis (C3GN3) demonstrated an improvement in kidney function with the creatinine level falling from a peak of $2.0 \mathrm{mg} / \mathrm{dL}$ to a new baseline of $1.4 \mathrm{mg} / \mathrm{dL}$ by the end of Eculizumab therapy. The posttreatment renal biopsy did not show any significant change as both pre- and post-treatment samples demonstrated mild mesangial proliferation with no endocapillary proliferation or exudative features. This patient's laboratory values remained unchanged at four and eight weeks after completion of therapy.

In summary of this Eculizumab trial, two patients (DDD1 and C3GN3) demonstrated improvements in serum creatinine levels, one patient (DDD3) experienced partial remission of his nephrotic syndrome, and one patient (C3GN2) demonstrated stable laboratory values throughout treatment and less proliferation on post-treatment renal biopsy. However, the other two patients in this study experienced declining renal function in spite of Eculizumab therapy. None of the patients enrolled in this clinical trial experienced any adverse effects or infections after Eculizumab therapy for the duration of the study. The results presented here suggest that Eculizumab shows great promise as an effective and appropriate treatment for a subgroup of patients with dense deposit disease and C3 glomerulonephritis [5].

Daina et al. [16] case reported of the case of an 11-year-old patient with nephrotic syndrome (urinary protein excretion of $4.8 \mathrm{~g}$ per twenty hours) and an original diagnosis of membranoproliferative glomerulonephritis. With this diagnosis, she was treated with glucocorticoids for five years with no significant improvement in her clinical status. Glucocorticoids were withdrawn and the patient was started on ramipril and losartan with no improvement of proteinuria. At this point in time another biopsy was performed and the diagnosis of dense deposit disease was made. The patient was treated with rituximab which similarly did not improve her nephrotic-range proteinuria. After these failed treatments, the patient was started on Eculizumab at a dose of 900 mg weekly for five and then $1200 \mathrm{mg}$ every 2 weeks. Before initiation of Eculizumab, her urinary protein level was $5.42 \mathrm{~g} / \mathrm{dL}$ and serum creatinine was $2.2 \mathrm{mg} / \mathrm{dL}$. At a forty-eight week follow-up, her serum total protein and albumin had normalized, creatinine levels had decreased, and her proteinuria had declined to below the nephrotic range with no report of any side-effects or complications.

Vivarelli et al. [17] presented a promising case study of a young female patient who was diagnosed with dense deposit disease. Throughout the course of disease, the patient experienced relapsing proteinuria, microhematuria and low plasma C3 levels. After seven years of the onset of disease, the patient's proteinuria greatly increased with a urinary protein: creatinine ratio of 2 to 3 . A renal biopsy taken at this time revealed focal sclerosis in $40 \%$ of glomeruli. Before initiating therapy with Eculizumab, the patient was taking ramipril and losartan. These medications were maintained as trial with Eculizumab was started. Quickly after initiation of the Eculizumab, the patient's proteinuria decreased and plasma protein levels were increased. Her microhematuria also decreased to trace levels. Repeat renal biopsies were performed at six and eighteen months after therapy with eculizumab was started. Both biopsy specimens demonstrated progressive reduction in mesangial proliferation as well as a reduction in the thickness of glomerular capillary loops. Dense deposits also appeared to be reduced in the biopsy taken at 18 months as seen by electron microscopy. Although the biopsy findings were highly encouraging, the progression of tubular atrophy and glomerular sclerosis was observed [17]. Eculizumab was discontinued in this patient at 18 months and resulted in rapidly increasing proteinuria. Therapy was resumed 6 months later and the patient's proteinuria decreased. This patient's improved lab results and repeat renal biopsies show that eculizumab does in fact have an effect in dense deposit disease.

Mcaughan et al. [13] present the case of a 29-year-old woman who began Eculizumab therapy after having received a renal transplant from her brother. She had been diagnosed with dense deposit disease at the age of 7 . 
Her disease progressed to end stage renal disease sixteen years after diagnosis and she had been treated with peritoneal and hemodialysis in the six years preceding renal transplantation. Four weeks after a successful transplantation, the patient developed proteinuria (equivalent to $6 \mathrm{~g} / \mathrm{dL}$ in $24 \mathrm{hrs}$ ), and her serum creatinine was 1.4 $\mathrm{mg} / \mathrm{dL}$. At five weeks post-transplant, the patient underwent a renal biopsy of the transplant and the specimen demonstrated cellular crescents and neutrophil polymorphs in the glomeruli with endocapillary proliferation. Strong linear C3 deposition along the glomerular basement membrane and within the mesangium was revealed by immunofluoresence. These findings were consistent with a recurrence of her dense deposit disease. With this diagnosis confirmed, the patient was treated with steroids, plasmapheresis, and anti-CD20 monoclonal antibody (rituximab), all to no avail. The patient's renal function continued to deteriorate with her creatinine rising to 4.93 $\mathrm{mg} / \mathrm{dL}$. She developed hypertension and was determined to be clinically nephrotic. Eculizumab was started in this patient thirteen weeks post-transplant in response to her declining kidney function. She received two $900 \mathrm{mg}$ loading doses each one week apart and then was continued with maintenance therapy of $600 \mathrm{mg}$ once every other week. Immediately after starting Eculizumab, the patient showed a significant improvement in both her clinical status and laboratory values. Her creatinine decreased by $1.9 \mathrm{mg} / \mathrm{dL}$ during only the first two weeks of therapy and her urine protein levels also significantly improved [13]. Also, there were no drug therapy related side effects or complications associated with the drug use. Although there is no follow-up biopsy from this case, the patient's lab values and clinical status positively support the effective role of Eculizumab in this patient's dense deposit disease.

Given the diversity of $\mathrm{C} 3$ glomerulopathy and the various mechanisms by which the alternative complement pathway is over-activated, it is not surprising that these presented patients demonstrated variable responses to Eculizumab. The trial performed by Bomback et al. presented four patients that showed various improvements in renal function and/or clinical status after therapy with Eculizumab. However those responses were very different and non-uniform, providing inconclusive data as to the exact mechanism of Eculizumab in different patients. Although the results appear positive, this trial also only consisted of six patients which is an extreme limitation to this study [5]. But despite the small subject size study of Bomback et al., the positive patient responses are reassuring that Eculizumab does have a role to play in $\mathrm{C} 3$ glomerulopathy. This study creates motivation and reason for the community of nephrology research to delve further into the benefits and uses of Eculizumab for $\mathrm{C} 3$ glomerulopathy patients. The various case studies reported above also point to Eculizumab as a treatment to be seriously considered. The three separate case reports demonstrate individual patients with different presentations of $\mathrm{C} 3$ glomerulopathy that all respond very positively to Eculizumab. However two of these reports lack post-treatment biopsy which is a limitation. The small number of individual patient case reports is another limiting factor in declaring Eculizumab as an appropriate therapy for $\mathrm{C} 3$ glomerulopathies. However, with the reported data here, it is safe to state that Eculizumab has earned the right to be properly researched with more comprehensive patient pools and clearer study designs with appropriate follow-up. The data here support and confirm that Eculizumab has a positive role to play in the management of $\mathrm{C} 3$ glomerulopathy.

\section{Conclusion and Future Recommendations}

A group of experts in renal pathology, nephrology, complement physiology and complement therapeutics held a conference in 2012 to discuss C3 glomerulopathy and delineate the definitions and recommendations for diagnosis and management of this disease [11]. Anti-complement therapy is the logical choice, but there are still quite a few questions that require answers and further research upon. Is it possible to achieve better remission with standard anti-cellular immunosuppressives, or inhibitors of the anaphylatoxins in the future? Who are the patient candidates that should be treated by this methodology? Is the response to therapy predictable and reproducible over a larger number of patients? When is it an ideal time to start the therapy and how long should it last? It is obvious that further research is necessary to differentiate phenotypes, characterize laboratory patterns, including pathohistological findings, and assess genetics background to optimize clinical trial design. As discussed in the paper, the existing case studies and small clinical trial dealing with Eculizumab in $\mathrm{C} 3$ glomerulopathy patients suggest a positive pharmaceutical affect. Many of the patients demonstrated in the case studies experienced an extreme clinical improvement after administration of Eculizumab. Many of these patients had exhausted prior treatment options including calcineurin inhibitors, plasmapharesis, and anti-CD20 monoclonal antibodies with no affect. Eculizumab served as a last resort and as such, the positive result seen in these patients is an important landmark in the research of $\mathrm{C} 3$ glomerulopathy. But due to the limited number of patients, it is 
too early to declare Eculizumab as a success. Thus far we can only strongly suggest that it is a viable treatment option. Although the current medical evidence regarding this medication is still insufficient to make a sure claim, results do provide the medical community with a great source of motivation to immediately investigate further. Much more organized research with larger patient populations is needed to continue studying the effects of Eculizumab on the pathophysiology of C3 glomerulopathy and what role it has to play in maintenance and treatment of patients who are currently left with little or no other options.

\section{References}

[1] Fakhouri, F., Frémeaux-Bacchi, V., Noël, L.-H., Cook, H.T. and Pickering, M.C. (2010) C3 Glomerulopathy: A New Classification. Nature Reviews Nephrology, 6, 494-499.http://dx.doi.org/10.1038/nrneph.2010.85

[2] Bomback, A.S. and Appel, G.B. (2012) Pathogenesis of the C3 Glomerulopathies and Reclassification of MPGN. Nature Reviews Nephrology, 8, 634-642. http://dx.doi.org/10.1038/nrneph.2012.213

[3] Nasr, S.H., Satoskar, A., Markowitz, G.S., Valeri, A.M., Appel, G.B., Stokes, M.B., Nadasdy, T. and D’Agati, V.D. (2009) Proliferative Glomerulonephritis with Monoclonal IgG Deposits. Journal of the American Society of Nephrology, 20, 2055-2064. http://dx.doi.org/10.1681/ASN.2009010110

[4] Sethi, S., et al. (2011) Proliferative Glomerulonephritis Secondary to Dysfunction of the Alternative Pathway of Complement. Clinical Journal of the American Society of Nephrology, 6, 1009-1017. http://dx.doi.org/10.2215/CJN.07110810

[5] Bomback, A.S., et al. (2012) Eculizumab for Dense Deposit Disease and C3 Glomerulonephritis. Clinical Journal of the American Society of Nephrology, 7, 748-756. http://dx.doi.org/10.2215/CJN.12901211

[6] Appel, G.B., et al. (2005) Membranoproliferative Glomerulonephritis Type II (Dense Deposit Disease): An Update. Journal of the American Society of Nephrology, 16, 1392-1403. http://dx.doi.org/10.1681/ASN.2005010078

[7] Habib, R., Gubler, M.-C., Loirat, C., Ben, H. and Levy, M.M. (1975) Dense Deposit Disease: A Variant of Membranoproliferative Glomerulonephritis. Kidney International, 7, 204-215. http://dx.doi.org/10.1038/ki.1975.32

[8] Sethi, S., Fervenza, F.C., Zhang, Y., Zand, L., Vrana, J.A., Nasr, S.H., Theis, J.D., Dogan, A. and Smith, R.J.H. (2012) C3 Glomerulonephritis: Clinicopathological Findings, Complement Abnormalities, Glomerular Proteomic Profile, Treatment, and Follow-Up. Kidney international, 82, 465-473. http://dx.doi.org/10.1038/ki.2012.212

[9] Nester, C.M. and Smith, R.J. (2013) Treatment Options for C3 Glomerulopathy. Current Opinion in Nephrology and Hypertension, 22, 231-237. http://dx.doi.org/10.1097/mnh.0b013e32835da24c

[10] Odum, J., et al. (1994) Recurrentmesangial IgA Nephritis Following Renal Transplantation. Nephrology Dialysis Transplantation, 9, 309-312.

[11] Pickering, M.C., et al. (2013) C3 Glomerulopathy: Consensus Report. Kidney International, 84, 1079-1089. http://dx.doi.org/10.1038/ki.2013.377

[12] Pickering, M.C., Cook, H.T., Warren, J., Bygrave, A.E., Moss, J., Walport, M.J. and Botto, M. (2002) Uncontrolled C3 Activation Causes Membranoproliferative Glomerulonephritis in Mice Deficient in Complement Factor H. Nature Genetics, 31, 424-428. http://dx.doi.org/10.1038/ng912

[13] McCaughan, J., O’Rourke, D. and Courtney, A. (2012) Recurrent Dense Deposit Disease after Renal Transplantation: An Emerging Role for Complementary Therapies. American Journal of Transplantation, 12, 1046-1051. http://dx.doi.org/10.1111/j.1600-6143.2011.03923.x

[14] Hillmen, P., et al. (2004) Effect of Eculizumab on Hemolysis and Transfusion Requirements in Patients with Paroxysmal Nocturnal Hemoglobinuria. New England Journal of Medicine, 350, 552-559. http://dx.doi.org/10.1056/NEJMoa031688

[15] Nürnberger, J., et al. (2009) Eculizumab for Atypical Hemolytic-Uremic Syndrome. New England Journal of Medicine, 360, 542-544. http://dx.doi.org/10.1056/NEJMc0808527

[16] Daina, E., Noris, M. and Remuzzi, G. (2012) Eculizumab in a Patient with Dense-Deposit Disease. New England Journal of Medicine, 366, 1161-1163. http://dx.doi.org/10.1056/NEJMc1112273

[17] Vivarelli, M., Pasini, A. and Emma, F. (2012) Eculizumab for the Treatment of Dense-Deposit Disease. New England Journal of Medicine, 366, 1163-1165. http://dx.doi.org/10.1056/NEJMc1111953 